\title{
APLICACIÓN DE LA REALIDAD AUMENTADA A LOS PROCEDIMIENTOS ORTODÓNTICOS
}

\section{ENFORCEMENT PROCEDURES AUGMENTED REALITY ORTHODONTIC}

\section{RESUMEN}

La irrupción de las Tecnologías de la información (TIC) enriquecen el proceso enseñanza-aprendizaje; el uso de los ambientes en realidad aumentada como apoyo a los procedimientos odontológicos mediante la utilización de procesos educativos en la preclínica de ortodoncia surge como alternativa para mejorar la práctica de los estudiantes en diferentes rutinas. Objetivo general: Diseñar, implementar y evaluar una aplicación de la realidad aumentada como apoyo a los procedimientos odontológicos, mediante la utilización de procesos educativos en la preclínica de ortodoncia. Materiales y métodos: La investigación se realizará en tres fases: fase 1: Se hará el estudio diagnóstico y diseño de la propuesta pedagógica donde Se contemplaran las necesidades de contar con ésta estrategia, los participantes a los que va dirigida la aplicación de la realidad aumentada, los Conocimientos previos con los que debe contar el usuario de la aplicación. La fase 2: Corresponde al desarrollo de la aplicación, en esta se analizaran los requerimientos de información sobre el tema, el diseño de la aplicación y se elaborará el prototipo para ser evaluado interna y externamente para aprobación definitiva. Fase 3: Denominada de Implementación y evaluación definitiva, se aplicará un instrumento de validación en prueba de campo así: La evaluación de contenidos académicos se realizará siguiendo el diseño pos prueba y grupo control, en el que se seleccionará al azar un grupo control y un grupo experimental, con el propósito de manipular la variable independiente poniendo en práctica el principio de presencia ausencia y observando el comportamiento de la variable dependiente. Aplicando la estrategia didáctica propuesta se determinará si esta es una herramienta más eficaz que el método de enseñanza tradicional para la enseñanza de los contenidos relacionados con doblaje de alambres en ortodoncia. Los resultados esperados de la investigación son: Una aplicación en ambiente de realidad aumentada como apoyo a los procedimientos odontológicos, mediante la utilización de procesos educativos en la preclínica de ortodoncia.

Palabras clave: Ortodoncia, Realidad aumentada, Tecnología educativa.

\section{ABSTRACT}

The emergence of Information Technology (ICT) enrich the teachinglearning process; the use of augmented reality environments to sup-
Jaime Alberto Paez Paez:

Ingeniero de Sistemas. Maestría en Educación-Universidad Pedagógica Nacional-Colombia. Docente Universidad Cooperativa de Colombia - correo: Jaime.paez@campusucc.edu.co Jairo Augusto Cortes Mendez: Ingeniero de Sistemas Master en Sociedad de la Información y el Conocimiento-Universidad Oberta de $\mathrm{Ca}$ talunya-España. Master en Dirección Universitaria-Universidad de los Andes. Doctorado Universidad Oberta de Catalunya-España.

Docente Universidad Cooperativa de Colombia- correo: Jairo.cortes@campusucc.edu.co

Maria Alejandra Gonzalez Bernal: Odontóloga-Universidad el BosqueEscuela Colombiana de Medicina-Colombia. Master en Administración de Salud-Universidad Pontificia Javeriana-Colombia.

Docente Universidad Cooperativa de Colombia-correo: m_alejandragonzales@yahoo.com

\section{Adiela Ruiz Gómez:}

Odontóloga-Universidad Nacional de Colombia-Colombia. Bióloga y Química-Universidad Pedagógica Nacional-Colombia. Especialista en Epidemología-Universidad de Antioquia-Colombia.

Docente Universidad Cooperativa de Colombia- correo: adielaruizo@hotmail.com

Tipo: Reporte de Caso

Fecha de Recepción: 04-Abr-2015 Fecha de Aceptación: 24-Jun-2015 
port dental procedures through the use of educational processes in preclinical orthodontic emerges as an alternative to improve the practice of students in different routines.

Objective: To design, implement and evaluate an application of augmented reality to support dental procedures through the use of educational processes in preclinical orthodontic. Materials and Methods: The research was carried out in three phases: Phase 1: the diagnostic study and design of the pedagogical approach where the needs of having contemplate this strategy will be made, the participants who are targeted in the implementation of augmented reality, previous knowledge with which the user must have the application. Phase 2: It is the application development in this reporting requirements on the subject was analyzed, the application design and prototype to be evaluated internally and externally to develop final approval. Phase 3: Implementation Called and definitive assessment, an instrument validation test will be applied in the field as follows: Evaluation of academic content will be made following the design posttest and control group, in which randomly selected control group and a experimental group with the purpose of manipulating the independent variable implementing the principle of presence and absence observing the behavior of the dependent variable. Applying the teaching strategy proposal will determine whether this is a more effective tool than traditional teaching method for teaching content related to dubbing orthodontic wires. The expected results of the investigation are: An application in augmented reality environment to support dental procedures, using educational processes orthodontic preclinical.

Keywords: Orthodontics, Augmented Reality, Educational Technology.

\section{INTRODUCCIÓN}

Los alambres son los principales elementos activos utilizados en el tratamiento ortodóntico, al ser doblados por el ortodoncista, transmitirán presión sobre los dientes lo que más tarde será convertido en respuesta biológica para producir el movimiento dentario [1].

En el ámbito educativo, las tecnologías de la información y la comunicación (TIC), están promoviendo una nueva visión del aprendizaje y del conocimiento, situación a la que no es ajena la odontología. La introducción de estas tecnologías en el proceso enseñanza-aprendizaje, brindan condiciones óptimas para transformar la enseñanza en otro tipo de educación donde el estudiante se hace más participativo en la apropiación del conocimiento, sin embargo, ellas por sí solas no garantizan el éxito [2]; entre las tecnologías que complementan la percepción e interacción con el mundo real se encuentra la realidad aumentada, la cual surge como respuesta a las necesidades, vivencias y emociones que el ser humano requiere para mejorar los niveles de interactividad con la tecnología, especialmente en la relación de lo real con lo virtual y viceversa.

Todavía se estudia el grado de efectividad de esta tecnología en los infinitos campos de aplicación que tiene; en ella "la cámara captura la información del mundo real, el sistema de posicionamiento determina la ubicación y orientación del usuario en cada momento, con esta información se genera el escenario virtual que se va a mezclar con la señal de video capturada para generar la escena aumentada. Esta escena compuesta por la información real y la virtual, se presenta al usuario a través del dispositivo de visualización (dispositivos móviles, visor de imagen digital)".

En la especialización de ortopedia funcional y ortodoncia de la Universidad Cooperativa de Colombia, sede Bogotá, se imparte una preclínica en ortodoncia, en donde se enseña el doblaje de alambres, allí los estudiantes desarrollan habilidades a partir de la instrucción teórica y práctica con ejercicios activos, no obstante, las demostraciones prácticas se hacen en un tiempo de trabajo breve, lo cual hace que el estudiante cuando requiera repetir este procedimiento no cuente con una estrategia didáctica que le permita recrear esta práctica aunque cuente en la especialización con los CD 
sobre biomecánica [3], alambres en ortodoncia [4] y dobleces en ortodoncia [5], que le permiten repasar la teoría pero no el acceso a repetir el proceso en el que se sientan inseguros. Actualmente, se reportan investigaciones sobre la utilización de esta tecnología en ciencias de la educación, ejemplo de ello, es el trabajo realizado por Basogain X, Olabe M, Espinosa K, Rouèche $\mathrm{C}$ y Olabe JC, sobre realidad aumentada en la educación: una tecnología emergente donde se describe el fundamento básico de la misma y se enumeran diferentes iniciativas de su aplicación en diferentes ámbitos de la educación, quedando fuera del alcance del artículo los aspectos pedagógicos y tecnológicos que conlleva la Realidad Aumentada [6].

Una de las aplicaciones más populares de Realidad Aumentada en la educación, es el proyecto Magic Book, del grupo HIT, de Nueva Zelanda. En este proyecto, el usuario puede leer un libro mediante un visualizador de mano y acceder a contenidos virtuales. Cuando en una página encuentra información interesante, el usuario puede introducirse y experimentar la escena en un entorno inmersivo [7].

En cuanto a la aplicación de la realidad aumentada en odontología, la búsqueda bibliográfica arroja pocos estudios, entre ellos está el desarrollado por Alemán JA, sobre Odontología Robótica en Odontosalud - Video 2 en donde desde la realidad aumentada se capacita sobre la colocación de un implante dental [8].

En La Universidad Cooperativa de Colombia (UCC), sede Bogotá, se ha venido trabajando en la consolidación de un modelo pedagógico mediado por las TICs a través de un portafolio de contenidos virtuales que hasta el momento le permite contar con 60 mil estudiantes- materia que en los últimos cuatro años han tomado los cursos institucionales en la modalidad virtual [9]. Las especializaciones de odontología en la sede Bogotá, han venido organizando una serie de software educativos y cursos virtuales como apoyo educativo a través de trabajos de grado y docentes de la mano del grupo de investigación ODONTOPOSTGRADOS UCC, no obstante al ser analizados resultan con deficiencias que ame- ritan una reestructuración desde la tecnología educativa con el fin de perfeccionarlos y encaminarlos hacia las nuevas tecnologías, es así como surge la propuesta sobre el uso de la realidad aumentada en la enseñanza de dobleces en alambre como una alternativa para mejorar la practica en la preclínica antes mencionada.

PREGUNTA DE INVESTIGACIÓN: ¿En la preclínica de ortodoncia la utilización de dos estrategias educativas sobre el doblaje de alambres: realidad aumentada y explicación verbal (tradicional) presencial mejora el tiempo en el doblaje de alambre y la habilidad manual sobre este procedimiento en el estudiante de ortopedia funcional y ortodoncia?

El Objetivo general de la investigación se formula como: Determinar la efectividad de la realidad aumentada como apoyo a los procedimientos de doblaje de alambres en la preclínica de ortodoncia, mediante la utilización de ésta en el proceso educativo. Lo Objetivos específicos son:

Establecer los contenidos temáticos sobre el doblaje de alambres en ortodoncia. Diseñar, una aplicación de la realidad aumentada como apoyo a los procedimientos de doblaje de alambres en la preclínica de ortodoncia. Implementar y evaluar la aplicación en los procesos cognitivos sobre el doblaje de alambres. Determinar la efectividad de la realidad aumentada vs clase tradicional en los procesos cognitivos sobre el doblaje de alambres

\section{MARCO TEORICO}

Los alambres en ortodoncia son hilos metálicos de acero inoxidable o de metal precioso de dimensiones y grados [10] con múltiples usos; en ortodoncia son utilizados para proveer las fuerzas que generan el movimiento dental; estas diferentes fuerzas que el alambre almacena en virtud de su posición y su composición son liberadas lentamente estimulando el ligamento periodontal produciendo cambios a nivel biológico, químico, celular y molecular permitiendo el movimiento del diente [11].

Se han utilizado como aditamentos para influir en la posición de los dientes, es así como desde 
el siglo XVIII con la invención del arco vestibular compuesto de una aleación de plata - níquel o platino - oro de grueso calibre 0.032 a 0.036 pulgadas los dientes eran ligados a este arco que servía como base para su alineación, sin embargo, producía movimientos de inclinación [12].

Hacia los años veinte Edward Angle invento los brackets de canto con metales preciosos como el oro y la plata alemana, siendo más flexibles, no obstante no cumplían todos los requisitos para realizar un tratamiento adecuado [13].

En la década de los treinta, aparecen los arcos de acero inoxidable de hierro, carbono, níquel y cromo con propiedades superiores a los métales preciosos en cuanto a modulo elástico, rango de trabajo y ductibilidad; en esa misma década, se crearon las tiras de acero inoxidable templado y se introdujeron los flujos de fluoruros permitiendo una soldadura eficaz. A la par de esto, el avance en la metalurgia, permitió crear alambres redondos, cuadrados, rectangulares y trenzados con variaciones controladas de dureza y resilencia. En los años 50 un nuevo alambre se presentó en ortodoncia con unas características similares al acero en cuanto a rigidez y los fabricaban en cuatro tipos de resilencias. El níquel Titanio creado por la marina americana en los años de 1960 por William Bühler fue utilizado en los años 70 por Andreasen G. quien lo utilizo como arcos para ortodoncia con el nombre de Nitinol.

Es de destacar que las propiedades ideales de un alambre en ortodoncia son: Biocompatibilidad, gran elasticidad y deflexión, estético, bioinerte, no adherente a placa bacteriana, económico, biocompatible, resistente a la corrosión y fractura además de una baja fricción superficial [14].

Como se mencionó anteriormente, los alambres de ortodoncia pueden estar compuestos de diferentes metales y aleaciones, entre los primeros están: oro, acero y en cuanto a aleaciones que los integran se puede contar con las de cromo-cobalto, Elgiloy, NiTi, TMA y NíquelTitanio superelástico entre otras.
Los movimientos en ortodoncia como por ejemplo los del tercer orden o troques son el resultado de las fuerzas generadas por los alambres [10], transmitidos al diente por el bracket; estos movimientos se producen en los tres planos del espacio $[11,12]$. Es característica de los alambres usados en ortodoncia la capacidad de almacenar y liberar energía, la cantidad de energía liberada por un alambre depende la deformación elástica que se haya conferido al alambre y el modulo elástico propio de cada alambre siendo mayor el del acero que el del TMA. Siendo este el caso que realizará en el estudio de aplicación de realidad aumentada donde solo se aplicará al torque dado por alambres de acero deformados elásticamente.

Se define torque como la rotación buco lingual de las raíces de un diente o grupo de dientes sobre sus ejes, por acción de un alambre cuadrado o rectangular, esta acción es una cupla, que se crea entre las paredes de la ranura del bracket y las aristas del alambre [12].

El torque en ortodoncia es sinónimo de momento de torsión en física, la unidad de medida del momento de torsión se da en gramos por milímetro $(\mathrm{g} / \mathrm{mm})$ o Newtons por milímetro $(\mathrm{N} / \mathrm{mm})$.

Existen dos tipos de torques según la dirección de la raíz, el positivo y el negativo, el positivo da una inclinación palatina o lingual a la raíz, mientras que el negativo se da cuando la raíz se inclina hacia vestibular. Además se clasifica según la cantidad de dientes en individual, continuo o progresivo; el primero de ellos cuando solo se inclina las raíces de un solo diente, mientras que el continuo se produce cuando se inclinan las raíces de un grupo de dientes, la misma cantidad de grados, se utiliza en los dientes anteriores y superiores, por último el progresivo se da cuando se inclinan las raíces desde los caninos hasta los molares, siendo mayor el grado de inclinación en los molares, y se crea dando una torsión en espiral hacia los molares [11].

El conocimiento y manejo de los torques en alambres de acero o TMA, es de vital importan- 
cia para el estudiante de la especialización de ortopedia funcional y ortodoncia, debido a que las técnicas modernas de arco recto donde los torques están incluidos en el bracket, en muchos casos no logran dar las inclinaciones adecuadas y se debe recurrir a torques aplicados con alambres de acero o TMA $[13,14]$.

A pesar de la escasa bibliografía encontrada, autores como Crengut et al [25] utilizaron las tecnologías de realidad virtual y aumentada en la simulación de tratamiento de prótesis dentales fijas por medio del proyecto VirDenT con la finalidad de aumentar la calidad del proceso educativo en las facultades de odontología, ayudando a los estudiantes a aprender cómo se preparan los dientes para restaurarlos con coronas de cerámica sin metal. Suebnukarn S, et al [26] realizaron la medición del proceso, el resultado y el rendimiento de prostodoncistas expertos y estudiantes aplicando un sistema de realidad virtual táctil en la preparación de una corona, reportaron que la comparación entre los grupos con respecto al rendimiento y la preparación de la corona fueron estadísticamente significativos $(\mathrm{p}<0.05)$. Ghanai $S$ et al [27] desarrollaron una aplicación de un articulador en 3D para simular el tratamiento en pacientes que requirieran cirugía ortognática, aunque el sistema puede reproducir el tratamiento previsto presenta fallas en el movimiento del paciente en la toma de rayos $\mathrm{X}$, en la ubicación de los puntos utilizando diferentes modelos de yeso, y en la marcación de los puntos en las imágenes.

La Realidad Virtual es una técnica en la que se trata de lograr una inmersión de un individuo en un ambiente tridimensional generado por computador. Esta idea fue concebida en los años 60 por Ivan Sutherland que fue el primero en lograr la creación de un espacio "real", en el cual el usuario podría moverse y navegar de manera similar a la vida real. "Su idea inicial constaba de un casco que proyectaba el mundo sobre los ojos del observador, y que registraba los cambios en su posición para actualizar la vista del usuario"[29] .

Después de esto se hizo varios ajustes sobre lo ya implementado con el fin de hacer parecer más real esta técnica, básicamente lo que busca la realidad virtual es "engañar a los sentidos, de forma que se haga creer al usuario que se encuentra delante de una realidad, que sólo existe en la memoria de una máquina y no tiene correspondencia con ningún objeto material o existencia real"[30] . Gracias o los avances en las redes de computadores y la implementación del Internet se ha podido avanzar en estas áreas tecnológicas.

Fue entonces que en 1994 durante una Conferencia Europea sobre Web, Tim Berners-Lee hizo presentación de una propuesta que buscaba el modelado de mundos virtuales creando un lenguaje para dicho fin, que pudiera hacer los mundos virtuales navegables a través de la Internet. De ahí se deriva la realidad virtual no inmersiva, titulada así porque no le permite al usuario tener conexiones sensoriales con su implementación, sino que le brinda al usuario un mundo virtual el cual puede explorar a través de internet pero sin apartarse de la realidad.

Realidad Aumentada: El termino fue acuñado en el año 1992 por Caudell [15], y se diferenció de la realidad virtual solo hasta el año 1997 en el estudio que realizo Ronald Azuma [16], donde expone que la realidad aumentada es una variación de la realidad virtual, donde se sumerge al usuario en el mundo virtual con el real, de tal forma que este último está presente todo el tiempo, mientras que la realidad virtual el usuario no percibe el mundo real. Como ejemplo se plantea la película "¿Quién engañó a Roger Rabbit?" que es una mezcla entre los real $\mathrm{y}$ virtual.

\section{DISEÑO INSTRUCCIONAL}

El diseño instruccional: La teoría de David Merrill.

El modelo plateado por este pedagogo y su grupo de investigación está plasmado en el trabajo ID research group y el ID2 research group, el cual define una completa y detallada estructura de los modelos mentales de los alumnos en una 
institución; y como por medio de la instrucción basada en tecnológica, estos modelos mentales cambian llegando a que el alumno aprenda de manera más fácil y efectiva [17]. El modelo pedagógico contiene desde la forma en que un maestro logra apoyarse en recursos tecnológicos para hacer mejor su trabajo y realmente enseñar con buenos resultados, hasta una propuesta de las formas de aprendizaje de un individuo teniendo en cuenta todos los factores sociales, psicológicos, de relación interpersonal y el antiguo conocimiento adquirido que influyen en forma de aprender.

La primera generación del Diseño Instruccional (ID), fue basado casi en su totalidad en las teorías de Robert Gagné, para esta nueva formulación se han contemplado las nuevas tecnologías y las tendencias contemporáneas de la educación.

La segunda generación del Diseño Instruccional (ID2): Con ID2 se puede:

Analizar, representar y dirigir una metodología de instrucción para la enseñanza integrada de conocimientos y habilidades por medio de un sistema.

Producir las prescripciones pedagógicas para la selección de estrategias educacionales interactivas y la selección de un orden de transacciones educacionales fijas.

Incorporar nuevo conocimiento sobre la enseñanza y el aprendizaje y la aplicación de estos a diseño de procesos.

Integrar estas fases al diseño instruccional. ID2 abarca los siguientes componentes: Una base teórica que organiza conocimiento sobre diseño educacional y define una metodología para realizar diseño educacional Una base de conocimientos para representar el dominio de conocimiento para propósitos de tomar decisiones educacionales.

Una serie de herramientas de diseño computarizadas inteligentes para análisis y adquisición del conocimiento, estrategias de análisis y la generación y conFiguración de transacciones.
Una colección de mini-expertos, cada uno que contribuye una base de conocimiento pequeña, relevante a una decisión educacional particular del diseño o a un sistema de tales decisiones.

Una biblioteca de transacciones educacionales para la entrega de la instrucción, y la capacidad de agregar nuevas o existentes transacciones a la biblioteca.

Un programa inteligente en línea del consejero que modifica dinámicamente la instrucción para requisitos particulares durante entrega, basado en un diálogo de la mezclar-iniciativa con el estudiante.

La estructura general del ID2 está conformado por los siguientes elementos:

\section{RETROALIMENTACIÓN}

El ambiente donde se desarrolle el curso debe permitir:

Observación sobre tareas, progresos en forma individual y grupal

Estar en contacto con los estudiantes una vez por semana para evaluar el desarrollo del curso

\section{EVALUACION}

Una etapa de todo proceso pedagógico está en la evaluación por eso se deben establecer:

Tareas específicas, Fechas de entrega, Criterios de evaluación sobre las siguientes estrategias: Tareas, proyectos, participación, moderación, exposición de trabajos en red, examen.

CARACTERIZACION DEL INSTRUCTOR

El tutor instructor debe tener habilidades en el uso de plataformas tecnológicas en especial tener:

Destreza de trabajo en red, Conocimientos del Software, Manejo de Listas de FAQs, Identificar a los estudiantes tutores, Conferencias en línea CARACTERIZACION DEL ESTUDIANTE

El estudiante debe tener unos mínimos conocimientos sobre la red, internet, en particular sobre la:

Posibilidad de Conectarse a la red, saber cómo solicitar ayuda, Aprender correo electrónico de la plataforma, Aprender a subir y bajar archi- 
vos, Ser proactivo.

\section{COMPONENTES DE LA PLATAFORMA}

El ambiente virtual debe tener la Infraestructura Tecnológica adecuada para que se pueda desarrollar el curso, por eso esta como mínimo contempla lo siguiente:

Correo electrónico: Se pueden desarrollar tutorías estableciendo comunicación entre estudiantes y profesores

Correo electrónico: Distribución cerrada de correo como soporte para distribuir materiales o noticias para cada uno de los inscritos en el curso

Chat : Comunicación sincrónica para la interacción real entre estudiantes

Foros de Discusión: Debate sobre problemas planteados entre estudiantes y profesores Contenido: Espacio donde se desarrollan las unidades temáticas.

\section{METODOLOGIA (MATERIALES Y METODOS)}

La metodología para el desarrollo de la Investigación será: Tipo de Estudio es un ensayo clínico aleatorizado. Población Estudiantes de la especialización de ortopedia funcional y ortodoncia.

Criterios de Selección: Inclusión que asistan a las preclínicas de ortodoncia. Y deseen participar en el estudio. Exclusión que sean estudiante repitentes en la preclínica de ortodoncia.

Muestra::Muestreo Aleatorio simple.

Hipótesis:

H0 La efectividad de la aplicación de la realidad aumentada como apoyo a los procedimientos de doblaje de alambres en preclínica de ortodoncia es igual a la efectividad de los procedimientos de doblaje de alambres explicados en forma tradicional en la preclínica de ortodoncia HA La efectividad de la aplicación de la realidad aumentada como apoyo a los procedimientos de doblaje de alambres en preclínica de ortodoncia es diferente a la efectividad de los procedimientos de doblaje de alambres explicados en forma tradicional en la preclínica de ortodoncia
Procedimiento:

La investigación se realizará en tres fases:

Fase 1. Diagnóstico de necesidades: Se utilizará Las técnica de grupo focal[19] con los estudiantes de la preclínica de ortodoncia para conocer acerca de cuál información sobre doblaje de alambres sería la más indicada de desarrollar en una estrategia educativa que utilice la realidad aumentada. Se analizaran aspectos como: Dificultad en el aprendizaje del doblaje de alambres, temas de difícil comprensión, dificultad en el desarrollo de talleres sobre doblaje de alambres, identificación de los problemas más frecuentes en el doblaje de alambres que permitan ser solucionados con el apoyo de dicha herramienta; el proceso de análisis de los datos de este grupo focal, permitirá la organización de los mismos en categorías de análisis que servirán como base para priorizar el tema sobre doblaje de alambres que se organizará en una o varias unidades temáticas para el diseño y desarrollo de la herramienta de realidad aumentada.

Fase 2. Diseño y desarrollo de la herramienta de realidad aumentada. Elementos para el desarrollo de un proyecto con realidad aumentada.

Para poder hacer uso de la realidad aumentada se necesitan una serie de elementos y dispositivos. Los imprescindibles son los siguientes[20]: Monitor: es el elemento básico dónde se muestra la mezcla de realidad y componentes virtuales. Hay distintos tipos de monitores que van desde los más futuristas a los más convencionales.

Cámara: dispositivo que toma la información del mundo real y la transmite al monitor. En el mayor número de casos, los elementos virtuales dependen de los elementos físicos, es por eso que se usaran cámaras web para poder transmitir la imagen al monitor. En el resto de casos, será la propia vista la que jugará este papel.

Software: los programas son los que toman los datos reales y los transforma en realidad au- 
mentada. Hay distintos programas que permiten trabajar con realidad aumentada, uno de ellos muy conocido es Flash. Otros programas disponibles son:ARviewer: editor de libre distribución que permite crear aplicaciones fácilmente integrables en dispositivos con sistema operativo Android

ARToolkit: librería muy extensa de software para desarrollar aplicaciones de realidad aumentada.

D.A.R.T: sistema de programación que ayuda a añadir a los software la funcionalidad de visualizar la mezcla de objetos reales y virtuales. Además incorpora librerías de acciones ante estímulos que permiten seguir a objetos con un marcador en un video y reaccionar con información a tiempo real según sea su marcador.

Marcadores: los marcadores básicamente son imágenes de símbolos que el software interpreta y de acuerdo a un marcador específico realiza una respuesta específica. Es un recurso muy utilizado y que se está imponiendo actualmente. Hay diversos tipos de marcadores, los más comunes son los llamados marcadores de códigos matriciales. Este tipo de códigos no fueron diseñados para el uso en la realidad aumentada, si no que fueron inventados para ser el equivalente al código de barras. Se decidió crear, porque el código de barras al ser leído genera una serie de números que se buscan en una base de datos para relacionarlo con el producto. Pero el problema reside en que hay muchos productos y sus números pueden repetirse de código mundialmente. Un código QR es un código matricial, es un código de barras de dos dimensiones desarrollado en Japón en 1994 por Denso-Wave[21] para el seguimiento de los repuestos en la producción de automóviles. El código es capaz de almacenar entre 1.800 y 7.000 caracteres, según la codificación y redundancia incorporada para corregir errores.

\section{MÉTODOS DE VISUALIZACIÓN}

Los métodos de visualización de la realidad aumentada y la virtual se parecen bastante. Ambas necesitan un dispositivo para poder visualizar los elementos virtuales. En el caso de la RA[22], el monitor sería el dispositivo de uso más frecuente para poder mezclar realidad y ficción. Aunque cada día la lista es más larga incluyéndose muchos más dispositivos como pueden ser cascos (headsets), gafas, móviles (smartphones) e incluso displays espaciales. Sea cual sea el dispositivo de visualización, todos se rigen por el mismo esquema, el esquema de la RA[23].

Los dispositivos más usados para la visualización de la realidad aumentada son:

Headsets: los headsets son cascos o gafas (HMD Head-Mounted Display) compuestos de pantallas de cristal líquido que se colocan en frente de los ojos para que el software plasme en ellas las imágenes virtuales. Estas pantallas no son opacas, ya que deben dejar que la vista pueda apreciar la realidad física y a su vez que los elementos virtuales se puedan reproducir y verse como si estuvieran sobrepuestos.

Smartphones: Otra manera de poder experimentar la realidad aumentada es a través de los móviles de tipo Smartphone, ya que éstos poseen cámara, una pantalla y la posibilidad de descargar e instalar aplicaciones para el funcionamiento de la RA[24]. Aunque como se dijo en la introducción, la RA se está orientando mucho a proporcionar información en tiempo real al usuario. Este tipo de información suele ser acerca del lugar donde se encuentra, es por esto que además de los elementos mencionados anteriormente, también necesitará:

Displays espaciales: La Realidad Aumentada Espacial (SAR) hace uso de proyectores digitales para mostrar información gráfica sobre los objetos físicos. La principal ventaja es que el display no está asociado a ningún usuario y así pueden hacer uso de todos a la vez y trabajar en grupo, y también que no depende de la resolución de pantalla donde vaya a reproducirse. Además SAR permite al usuario no tener la necesidad de llevar equipo encima como los anteriormente mencionados, ya que gracias al proyector, la imagen virtual se puede reflejar en una pared, una mesa, o incluso en la propia piel. 
Fase 3. Para la implementación y evaluación, se seleccionara la muestra de estudiantes de preclínica de ortodoncia que cumplan con los siguientes criterios de inclusión: Inclusión que asistan a las preclínicas de ortodoncia. Y deseen participar en el estudio.

Se excluirán aquellos estudiantes que. Exclusión que sean estudiante repitentes en la preclínica de ortodoncia. Posteriormente, se hará la aleatorización de la muestra a través del programa Microsoft Excel en grupo 1 o experimental y grupo 2 o control. Antes de iniciar el experimento, el docente realizará un ejercicio sobre doblaje de alambres y calificará a cada uno de los estudiantes con una escala de Bueno (si el doblaje del alambre coincide exactamente con una plantilla elaborada para tal fin y que después corresponderá con la utilizada en la herramienta de realidad aumentada), regular (si a pesar de coincidir se encuentran errores de doblaje) y malo (si no coincide y presenta errores de doblaje), los resultados serán diligenciados en un formato Microsoft Excel elaborado para tal fin por los investigadores.

\section{Grupo Experimental 1:}

Los estudiantes recibirán durante la preclínica como apoyo al doblaje de alambres la herramienta de realidad aumentada, la que pueden consultar las veces que quieran. Al final del proceso el docente hará una evaluación practica sobre el procedimiento explicado en la clase a través de la herramienta y calificará como Bueno (si el doblaje del alambre coincide exactamente con el ejemplo de realidad aumentada), regular (si a pesar de coincidir se encuentran errores de doblaje) y malo (si no coincide y presenta errores de doblaje). Los estudiantes que lo hagan entre excelente y bueno recibirán un estímulo positivo (un punto de calificación en la clase que será acumulativo), a los estudiantes que se les califique malo, se les dará la oportunidad de repetir el procedimiento, pero sin ningún puntaje como estímulo.

\section{Grupo 2 o Control:}

Los estudiantes recibirán durante la preclínica como apoyo al doblaje de alambres la explicación verbal por parte del docente. Al final del proceso el docente hará una evaluación practica sobre el procedimiento explicado en la clase y calificará como Bueno (si el doblaje del alambre coincide exactamente con el ejemplo de realidad aumentada), regular (si a pesar de coincidir se encuentran errores de doblaje) y malo (si no coincide y presenta errores de doblaje). Los estudiantes que lo hagan entre excelente y bueno recibirán un estímulo positivo (un punto de calificación en la clase que será acumulativo), a los estudiantes que se les califique malo, se le dará la oportunidad de repetir el procedimiento explicándoles nuevamente el docente, pero sin ningún puntaje como estímulo. No se informará a los estudiantes que se trata de un experimento ni tampoco que serán evaluados. Todos tendrán el mismo número de sesiones (tres) y trabajaran con el mismo tipo de material, la única diferencia estará en la aplicación de las estrategias.

Terminada la fase de implementación y evaluación se procederá a hacer el análisis estadístico de los resultados, las variables cualitativas como sexo y doblaje de alambres serán analizadas con estadística descriptiva (porcentaje) y para la comparación de grupos se utilizará CHI cuadrado.

Aspectos Éticos: Según la resolución 008430 de 1998 se clasifica como una investigación sin riesgo.

Análisis estadístico: Las variables cualitativas se analizaran con estadística descriptiva (Porcentajes y proporciones, razones) y las Variables cuantitativas con promedio y desviación estándar. Para la comparación de las variables cualitativas se aplicara la prueba de Chi Cuadrado $\chi^{2}$.

\section{RESULTADO Y DISCUSION}

Con base en los resultados obtenidos en el grupo focal, se presentan algunas consideraciones que se deben tener en cuenta para el desarrollo de la estrategia de apoyo educativo basada en realidad aumentada para la preclínica de orto- 
doncia.

- Dificultades en el aprendizaje

El proceso enseñanza -aprendizaje en la preclínica de ortodoncia, se da en un escenario donde confluyen factores como la necesidad del estudiante de desarrollar y entrenarse en habilidades técnicas para aplicar en la clínica y la relación del alumno con el docente que guía el proceso; los participantes en el grupo focal plantean como problema la distribución del tiempo en la preclínica, aunque concuerdan la mayor parte de los estudiantes en que la habilidad del doblaje se relaciona con la práctica autónoma y la técnica para hacerlos; sin embargo, llama la atención que uno de ellos manifiesta que la dificultad de aprendizaje radica en el diseño y preparación de las clases; es de notar que la mayoría de los participantes mencionaron que les gustaría contar con más intensidad horaria en las preclínicas de ortodoncia.

- Inconvenientes en la elaboración de ansas y Figuras

En relación con los inconvenientes en la elaboración de ansas y Figuras los estudiantes manifestaron la gran dificultad en la confección de las ansas utilizadas en la alineación y nivelación especialmente en caja modificada, verticales con helicoide y ansas de cierre en T, se destaca lo expresado por algunos de los participantes quienes manifestaron la dificultad en hacer el torque, dobleces de Meaw y dobleces de tercer orden consecutivo; en la actividad del grupo focal, se evidencio una plantilla de Figuras para hacer los ejercicios que son dados por el docente de la preclínica, no se encontró una guía o texto que permitiera al estudiante comprobar el paso a paso en la elaboración de las ansas y/o Figuras; al ser esta una catedra teórico-práctica, es importante estar al tanto de que toda actividad propiamente humana, como la actividad práctica, exige el conocimiento teórico de lo que se va a realizar; hoy en día la enseñanza promueve que mediante esta, los alumnos se apropien de manera consciente de conceptos teóricos que les permitirán operar con base en ellos estableciendo nexos y relaciones sobre la temática tratada, lo cual favorecerá que el aprendizaje adquirido tenga significado y sentido [32].
De acuerdo con los resultados obtenidos se sugiere elaborar una guía didáctica que incluya un resumen de la teoría sobre ansas en ortodoncia y los pasos esenciales en la elaboración de ansas de alineación y nivelación.

- $\quad$ Sugerencias para mejorar la práctica Al ser indagados los estudiantes sobre sugerencias para mejorar la práctica el $80 \%$ de ellos estuvieron de acuerdo en tener alguna herramienta tecnológica para explicar los pasos para la elaboración de las ansas aunque no hubo seguridad sobre el tipo de herramienta a utilizar, algunos de los participantes en el grupo focal manifestaron la necesidad de contar con un tutorial o un video, solo cinco de ellos declararon no necesitar ayuda alguna.

\section{CONCLUSIONES}

Se aplicó la metodología de grupo focal para diagnosticar la necesidad de contar con una estrategia educativa mediada por las TIC como apoyo a la preclínica de ortodoncia.

Se identificaron las dificultades de aprendizaje, los inconvenientes en la elaboración de ansas y las sugerencias desde los estudiantes para mejorar las prácticas en la elaboración de ansas y Figuras en ortodoncia.

A partir de los resultados del grupo focal se definió el tema a tratar en la herramienta mediada por realidad aumentada que fue la comprobación de las ansas de alineación y nivelación.

Las recomendaciones desde el estudio se relacionan con la elaboración de una guía didáctica para la preclínica de ortodoncia, un video de pasos básicos para la elaboración de ansas de alineación y nivelación, así como una herramienta de comprobación en la elaboración de las ansas de alineación y nivelación mediada por la realidad aumentada. 
Referencias

[1] Bravo González LA. Manual de Prácticas de Ortodoncia. Universidad de Murcia. Secretariado de Publicaciones e Intercambio Científico. Impreso en PoblagráficSA. 1993.

[2] Delgado M. Arrieta X. Uso de las TICs en educación una propuesta para su optimización. Rev. Omnia. 2009; 15(3): 58-77.

[3] García C, Villareal L, Guzmán A. Diseño didáctico soportado en el uso de tecnologías de información y comunicación para la enseñanza de principios y conceptos de biomecánica en la formación de estudiantes de ortodoncia de la Universidad Cooperativa de Colombia.[Trabajo de grado Especialista en Ortopedia Funcional y Ortodoncia]. Bogotá: Universidad Cooperativa de Colombia, sede Bogotá. Programa Especialización 2007.

[4] Alzate J, Bastidas M, Grijalba C, Delgado M, Salamanca C. Recurso multimedia para la enseñanza de alambres en ortodoncia.[Trabajo de grado Especialista en Ortopedia Funcional y Ortodoncia]. Bogotá: Universidad Cooperativa de Colombia, sede Bogotá. Programa Especialización 2007.

[5] Collantes S, González J, Hernández M, Vanegas L. Diseño de un CD multimedia educativo de los diferentes dobleces en ortodoncia. [Trabajo de grado Especialista en Ortopedia Funcional y Ortodoncia]. Bogotá: Universidad Cooperativa de Colombia, sede Bogotá. Programa Especialización 2008.

[6] Basogain X,Olabe M, EspinosaK, Rouèche C,OlabeJC. Realidad Aumentada en la Educación: una tecnología emergente. Information and CommunicationsTechnology, ESIGELEC - Rouen, FranceElectrical and ComputerEngineering, CBU (USA),Escuela Superior de Ingeniería de Bilbao, EHUBilbao, Spainxabier. basogain@ehu.eshttp://multimedia.ehu. es.

[7] Pérez Trejo E. Nuevas aplicaciones multimedia en el ámbito de la educación a distancia (realidad aumentada). Boletín Sistema de Universidad Abierta y Educación a Distancia (SUAyED). Universidad Nacional Autónoma de México. (32) Jun-Jul 2011. Disponible en:Http://www.cuaed.unam.mx/boletin/ boletinesanteriores/boletinsuayed32/ multimedia.php

[8] Salmeron JI. Primera experiencia internacional del uso de Realidad Aumentada en ámbito de la implantología. Disponible en:http://www.google.com. $\mathrm{co} / \mathrm{url}$ ? sa $=\mathrm{t} \& \mathrm{rct}=\mathrm{j} \& \mathrm{q}=$ realidad $\% 20$ a umentada\% 20 en $\% 201$ a $\% 20$ educaci\%C3\%B3n\%20en\%20odont olog\%C3\%ADa\&source $=$ web \&cd $=8$ $\&$ cad $=$ rja $\&$ sqi $=2 \& v e d=0$ CFYQFjAH \&url=http $\% 3 \mathrm{~A} \% 2 \mathrm{~F} \% 2 \mathrm{Fwww}$.scoop. it $\% 2 \mathrm{Ft} \% 2 \mathrm{Frobo}$-tips $\% 2 \mathrm{Fp} \% 2 \mathrm{~F} 3335$ $753046 \% 2$ Fodontologia-robotica-enodontosalud-video-2\&ei=1GlHUbXuL4 uK8QSxz4DoAQ\&usg=AFQjCNEnMjik QYtbsODXQAktGQ3ekIje2w

[9] El Universitario Cesar Augusto Pérez González. Edit.mayo-junio 2012, ed. $\mathrm{n}^{\circ} 010$ ISSN 2027-7202.

[10] Nanda R. Biomecanicas y estética, estrategias en ortodoncia clínica-amolca, 2007.pag 1.

[11] Uribe R G. Ortodoncia teoría y clínica, CIB, 2004, pags 170.

[12] Kang B, Back S,Moh J, Yang W. Three dimensional relationship between the critical contac angle and the torque angle. AJODO 2003 -123, 64- 73.

[13] Gregoret J,Tuber E, Escobar 1. El tratamiento ortodóncico con arco recto, NM ediciones,pag 214-215.

[14] Bennett J,Mcllaughin R.Manejo ortodóncico de la dentición con el aparato preajustado. Isis medical. 1997. pag 56 $58-144$.

[15] AZUMA, R. (1997), "A Survey of Augmented Reality". En: Presence: Teleoperators and Virtual Environments, vol.6 ,nº 4 , pp. 355-385 http://citeseerx. 
ist.psu.edu/viewdoc/download?doi=10.1. $1.35 .5387 \&$ rep $=$ rep $1 \&$ type $=$ pdf [consultado: 26.02.2013].

[16] CAUDELL, T. P., MIZELL, D.W. (1992). "Augmented Reality: An Application of Heads-Up Display Technology to Manual Manufacturing Processes". En: International Conference onSystemSci ences,Kauai,Hawaii,vol.2,pp.659-669, http://ieeexplore.ieee.org/stamp/stamp. jsp? arnumber $=00183317$ [ consultado: 26.02.2013].

[17] D. Merril. Educational Technology, New York: Li \& Jones, 1991.

[18] IBM - RUP Rational Unified Process Herramientas para conFigurar RUP http:// www-306.ibm.com/software/ar/rational/ rup.shtml

[19] Formato Documento Electrónico (ABNT) BERTOLDI, Sandra; FIORITO, María Elisa; ALVAREZ, Mabel. Grupo Focal y Desarrollo local: aportes para una articulación teórico-metodológica. Ciencia, Docencia, Tecnología., Concepción del Uruguay, n. 33, nov. 2006 . accedido en 22 marzo 2013. Disponible

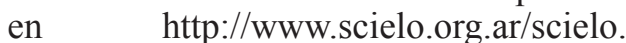
php? script $=$ sci arttext\&pid $=\mathrm{S} 1851$ $17162006000200005 \& \operatorname{lng}=\mathrm{es} \& \mathrm{nrm}=\mathrm{iso}$

[20] [Machado Marcos Carlos Aplicación de la realidad aumentada para un sistema de entrenamiento. Director: Pau Fonseca i Casas Junio 2011.http://upcommons.upc. edu/pfc/bitstream/2099.1/12455/1/73347. pdf

[21] Programa de Maestría en Ingeniería Civil (MS) Octubre 2012 http://www. techtear.com $/ 2008 / 03 / 28 /$ codigos-qr$\% \mathrm{C} 2 \% \mathrm{BF} q u e-$ son- $\% \mathrm{C} 2 \% \mathrm{BFpor}-q u e-$ existen-y-mas

[22] Theodoros N. Arvanitis James F. Knight Michael Gargalakos Human factors and qualitative pedagogical evaluation of a mobile augmented reality system for science education used by learners with physical disabilities. Received: 17 December 2006 / Accepted: 14 May 2007 / Published online: 20 November 2007 Springer-Verlag London Limited 2007

[23] Starner T, Mann S, Rhodes B, Levine J,
Healey J, Kirsch D, Picard R, Pentland A (1997) Augmented reality through wearable computing. Presence 6:386-398

[24] Kaufmann H, Schmalstieg D (2003) Mathematics and geometry education with collaborative augmented reality. Comput Graph 27(3):339-345

[25] Crengut, a M. Bogdana, Dorin $M$. Popovicia,b. Information system analysis of an e-learning system used for dental restorations simulation journal homepage : www.intl.elsevierhealth.com/journals/ cmpb

[26] Siriwan Suebnukarn a,*, Nattharat Phatthanasathiankul a, Sunantha Sombatweroje a, Phattanapon Rhienmora b, Peter Haddawy b. Process and outcome measures of expert/novice performance on a haptic virtual reality system. journal homepage: www.intl.elsevierhealth.com/ journals/jden

[27] S. Ghanai, R. Marmulla, J. Wiechnik, J. Mu"hling, B. Kotrikova: Computerassisted three-dimensional surgical planning: 3D virtual articulator: technical note. Int. J. Oral Maxillofac. Surg. 2010; 39: 75-82. \# 2009 International Association of Oral and Maxillofacial Surgeons. Published by Elsevier Ltd. All rights reserved.

[28] Ausubel D. teoría del aprendizaje significativo. Tomado de http://www. Educainformatica.com.ar/docentes/ tuarticulo/educación/Ausubel/index.html.

[29] Pedro M. Wightman, German A. Galvis, Daladier Jabba Ambientes de realidad virtual no inmersiva multiusuario con herramientas de software libre

[30] [30] MOLLÁ VALLÁ, Ramón. Realidad Virtual. Documentos de Clase. Universidad Politécnica de Valencia. 1999

[31] [31] http://www.maestrosdelweb.com/ editorial/que-es-realidad-aumentada/

[32] [32]Balzán Ballesteros JL. Estructura didáctica de la práctica profesional odontológica (relación objetivo contenidométodo). Educere. 2012; 16(54):113-30. 